\title{
Simplified DSP-Based Signal-Signal Beat Interference Mitigation for Direct-Detection Subcarrier Modulation
}

\author{
Z. Li, M.S. Erkılınç, R. Bouziane, R. Maher, L. Galdino, K. Shi, B.C. Thomsen, P. Bayvel, and R.I. Killey \\ Optical Networks Group, Department of Electronic \& Electrical Engineering, UCL (University College London), London, WC1E 7JE, UK \\ Authore-mail address: zhe.li@ee.ucl.ac.uk
}

\begin{abstract}
We proposed a novel SSBI mitigation technique with significantly lower DSP complexity. It was experimentally tested in $7 \times 25 \mathrm{~Gb} / \mathrm{s}$ WDM DD SSB 16-QAM N-SCM signal transmission over $480 \mathrm{~km}$ SSMF with a net ISD of $2.4(\mathrm{~b} / \mathrm{s}) / \mathrm{Hz}$.

OCIS codes: (060.0060) Fiber optics and optical communications; (060.2360) Fiber optics links and subsystems
\end{abstract}

\section{Introduction}

There is increasing demand for spectrally-efficient transmission systems using cost-effective optical transceivers in inter-data center, access, and metro links. For this purpose, direct detection (DD) subcarrier modulation (SCM) was recently proposed [1,2], and single sideband (SSB) Nyquist pulse shaped SCM (N-SCM) with high order QAM offers high information spectral density [3]. However, the signal-signal beat terms resulting from the square-law detection interfere with the desired signal-carrier beat products, an effect known as signal-signal beat interference (SSBI), causing a significant degradation in receiver sensitivity. It is desirable to develop effective techniques to mitigate SSBI, and consequently, improve the performance of DD SCM links.

Recently, an iterative digital post-compensation scheme has been demonstrated in [4,5]. However, the digital hardware complexity of the receiver is high due to the multiple iterations required. In this paper, we present a new SSBI mitigation technique, which combines pre-distortion and post-compensation and avoids the need for multiple iterations. We present experimental back-to-back and transmission results utilizing this technique in a WDM dispersion pre-compensated SSB 16-QAM N-SCM direct-detection system. SSBI compensation leads to $5.7 \mathrm{~dB}$ reduction in the required OSNR at the pre-FEC BER of $3.8 \times 10^{-3}$, and increases the achievable transmission reach.

\section{Simplified DSP-based Signal-Signal Beat Interference Mitigation}

As shown in Fig. 1, the simplified SSBI mitigation scheme has two steps: digital pre-distortion at the transmitter and receiver-based estimation and cancellation. The pre-distortion partially compensates the SSBI, improving the system performance, leading to better symbol decisions making, thus avoiding the requirement to perform multiple iterations in the post-compensation.

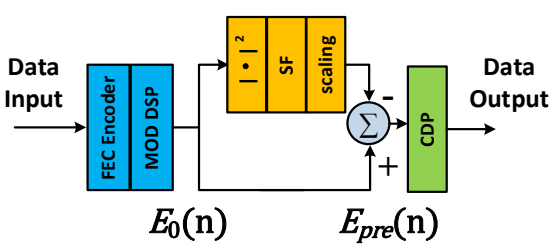

(a) Tx DSP

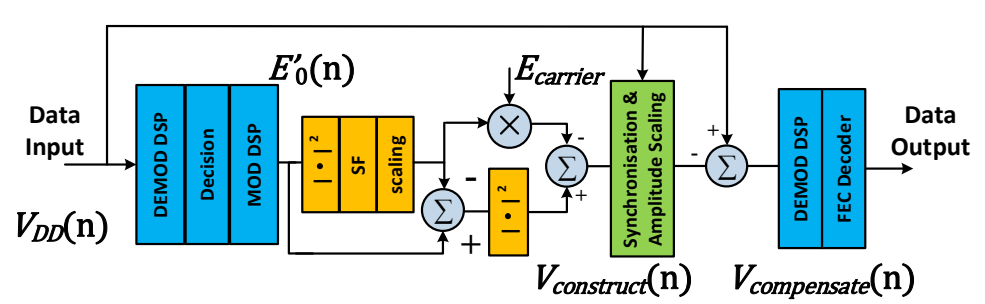

(b) Rx DSP

Fig. 1: (a) Transmitter DSP with SSBI pre-distortion and chromatic dispersion pre-compensation (CDP), (b) receiver DSP with SSBI postcompensation. MOD and DEMOD DSP: Modulation and demodulation DSP for single-sideband N-SCM signal. SF: sideband filter.

The principle of the pre-distortion is shown in Fig. 1(a): Following the generation of the SSB N-SCM signal by the modulation DSP, a digital representation of the signal-signal beating terms is calculated, which is subtracted from the original signal as follows:

$$
E_{p r e}(n)=E_{0}(n)-\eta \Lambda\left[\left|E_{0}(n)\right|^{2}\right]
$$

where $E_{\text {pre }}(n)$ is the sampled pre-distorted signal in the time domain, $E_{0}(n)$ is the SSB N-SCM signal after modulation DSP, $\eta$ is a scaling factor which is adjusted to optimize the performance. $\Lambda[\cdot]$ is the operator describing the transformation of the signal by the single sideband filter and $n$ is the discrete time index. Chromatic dispersion precompensation (CDP) is then performed to mitigate the dispersion of the transmission fiber. 
Following electrical-to-optical conversion with the addition of an optical carrier, $E_{\text {carrier }}$, transmission over the fiber and square-law detection, the detected signal $V_{D D}(n)$ is written as:

$$
\begin{aligned}
V_{D D}(n) & =\left|E_{\text {carrier }}+E_{\text {pre }}(n)\right|^{2} \\
& =E_{\text {carrier }}^{2}+2 \operatorname{Re}\left[E_{\text {carrier }} \cdot E_{0}(n)\right]+\left|E_{0}(n)\right|^{2}+\eta^{2} \cdot\left|\Lambda\left[\left|E_{0}(n)\right|^{2}\right]\right|^{2}-2 \eta \cdot \operatorname{Re}\left[E_{0}^{*} \cdot \Lambda\left[\left|E_{0}(n)\right|^{2}\right]\right]-2 \eta \cdot \operatorname{Re}\left[E_{\text {carrier }} \cdot \Lambda\left[\left|E_{0}(n)\right|^{2}\right]\right]
\end{aligned}
$$

In the right-hand side of this equation, the first term is simply the DC, which can be removed. The second term is the desired carrier-signal beating products, and the third to the sixth terms represent additional unwanted beating terms, which are relatively low due to the SSBI pre-distortion, and hence can be removed in a single step, rather than requiring the multiple iterative modulation and demodulation steps as used in [4]. As shown in Fig. 1(b), the detected signal waveform $\left(V_{D D}\right)$ is stored in memory, and then, demodulation DSP is performed. After making symbol decisions, a digital representation of the pre-distorted signal is re-generated and the effect of square-law detection is calculated. The beating terms between the carrier and signal-signal beating products are re-generated and subtracted from the detected signal waveform. Finally, the compensated signal is demodulated. The post-compensation technique is mathematically described as follows:

$$
\begin{aligned}
V_{\text {construct }}(n) & =\left|E_{0}^{\prime}(n)-\eta \Lambda\left[\left|E_{0}^{\prime}(n)\right|^{2}\right]\right|^{2}-2 \eta \operatorname{Re}\left[E_{\text {carrier }} \cdot \Lambda\left[\left|E_{0}^{\prime}(n)\right|^{2}\right]\right] \\
& =\left|E_{0}^{\prime}(n)\right|^{2}+\eta^{2} \cdot\left|\Lambda\left[\left|E_{0}^{\prime}(n)\right|^{2}\right]\right|^{2}-2 \eta \cdot \operatorname{Re}\left[E_{0}^{\prime \prime}(n) \cdot \Lambda\left[\left|E_{0}^{\prime}(n)\right|^{2}\right]\right]-2 \eta \cdot \operatorname{Re}\left[E_{\text {carrier }} \cdot \Lambda\left[\left|E_{0}^{\prime}(n)\right|^{2}\right]\right]
\end{aligned}
$$

where $V_{\text {construct }}(n)$ is the reconstructed beating terms, and $E_{0}{ }^{\prime}(n)$ is the re-generated SSB N-SCM signal based on the symbol decisions. After $V_{\text {construct }}(n)$ is subtracted from the stored signal waveform, since $E_{0}^{\prime}(n) \approx E_{0}(n)$, the compensated signal, $V_{\text {compensate }}(n)$ can be written as:

$$
V_{\text {compensate }}(n)=V_{D D}(n)-V_{\text {construce }}(n) \approx E_{\text {carrier }}^{2}+2 \operatorname{Re}\left[E_{\text {carrier }} \cdot E_{0}(n)\right]
$$

Therefore, the compensated signal only contains the DC term and the desired carrier-signal beating term, and the nonlinear distortion caused by square-law detection is reduced.

\section{Experimental Setup}

The optical transmission test-bed is shown in Fig. 2. Two IQ-modulators, with inputs from an optical comb generator (OCG), were used to generate odd and even channels. The modulators were driven by two DACs with a sampling rate of $25 \mathrm{GSa} / \mathrm{s}$. The modulation DSP generating the SSB N-SCM signal is described in detail in [3]: root-raised cosine (RRC, roll-off factor $\alpha=0.1)$ pulse-shaped 16QAM, at a symbol rate $\left(f_{s}\right)$ of 6.25 Gbaud encoded four decorrelated $2^{15}$ de Bruijn patterns. The signal was up-converted to a subcarrier frequency of $3.43 \mathrm{GHz}\left(0.55 \cdot f_{s}\right)$ and the lower frequency sideband was removed using a digital sideband filter. Finally, SSBI pre-distortion and CDP were carried out as shown in Fig. 1(a). The channel spacing of the WDM grid was set to $10 \mathrm{GHz}$.

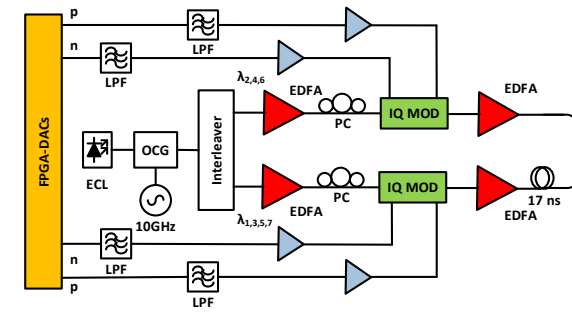

ECL: External cavity laser, OCG: Optical comb generator, DAC: Digital-to-analog converter, LPF: Low-pass filter, EDFA: Erbiumdoped fiber amplifier, PC: Polarization controller

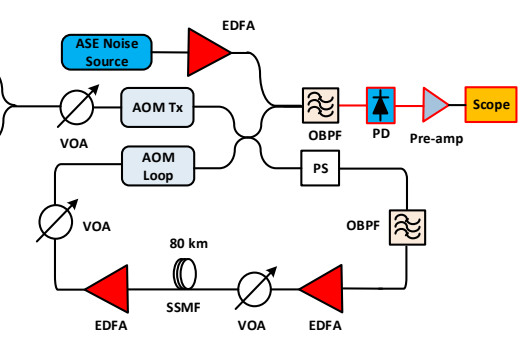

AOM: Acousto-optic Modulator, VOA: Variable optical attenuator, PS: Polarization scrambler, OBPF: Optical Band-pass filter, PD: Photodiode, SSMF: Standard single mode fiber, DSP: Digital signal processing
(I)

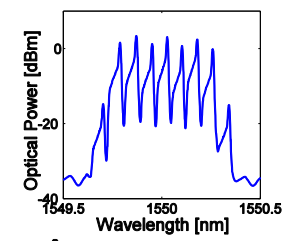

(II)

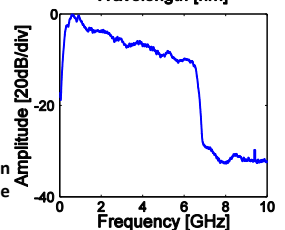

Fig. 2: Experimental setup. Inset: (I) Experimental WDM spectrum, (II) detected digital spectrum.

A recirculating fiber loop, with an $80 \mathrm{~km}$ span of standard single-mode fiber (SSMF), was employed to perform transmission. The total loss of $31 \mathrm{~dB}$ within the loop was compensated using two erbium-doped fiber amplifiers with $4.5 \mathrm{~dB}$ noise figure. At the receiver, an optical band-pass filter was used to demultiplex the channel of interest, which was detected using a single-ended PIN photodiode and digitized by a single ADC operating at $50 \mathrm{GSa} / \mathrm{s}$. Following the normalization and resampling to 2 samples per symbol, down-conversion and matched filtering were applied, respectively. A 5-tap CMA-LMS equalizer was used before switching to decision-directed LMS mode. After the hard decisions, the simplified SSBI post-compensation was applied to mitigate the SSBI, as described in the previous section (Fig. 1(b)). Finally, the BER was calculated. The proposed scheme was compared with the previously proposed 
receiver-based iterative SSBI cancellation scheme [5], setting the number of iterations to four for optimum performance.

\section{Results}

First, the system performance in back-to-back operation was assessed by ASE-noise loading at the receiver. The carrier-to-signal power ratio (CSPR) value was swept from $9 \mathrm{~dB}$ to $13 \mathrm{~dB}$ and adjusted at each OSNR level for the optimum performance. The experimental and simulated BER curves without SSBI compensation and with compensation (both simplified and iterative receiver-based) are plotted in Fig. 3(a). The proposed simplified SSBI compensation scheme provides similar performance to the more complex iterative cancellation technique. The constellation is significantly less distorted, particularly on the outer symbols, with the required OSNR (at BER = $3.8 \times 10^{-3}$ ) decreasing from $25.2 \mathrm{~dB}$ to $19.5 \mathrm{~dB}$. The optimum CSPR value using SSBI compensation was reduced by $3 \mathrm{~dB}$ compared to the uncompensated system [5]. Furthermore, the transmission performance of these two SSBI compensation schemes were compared by plotting the EVM versus the number of iterations for iterative SSBI compensation at distances of $240 \mathrm{~km}$ and $480 \mathrm{~km}$ (Fig. 3(b)). It can be seen that the iterative SSBI mitigation scheme requires multiple ( 3 or 4 ) iterations at the receiver to achieve optimum performance. However, the simplified SSBI mitigation scheme offers very similar performance while avoiding the need for multiple iterations.
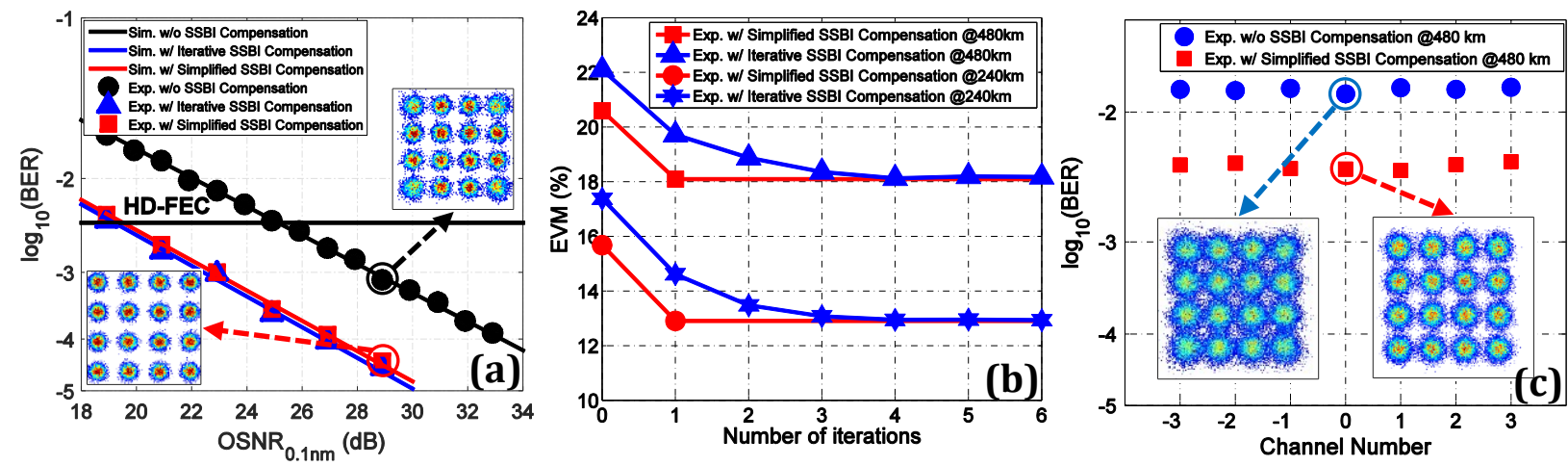

Fig. 3: (a) Back-to-back BER measurements. Inset: Received constellations without (EVM = 16.5\%) and with (EVM = 12.1\%) simplified SSBI compensation. (b) Comparisons of EVMs obtained using the simplified SSBI compensation scheme and iterative compensation. (c) BERs for each received channel after transmission over $480 \mathrm{~km}$; Inset: Received constellations for center channel without (EVM $=22.1 \%)$ and with $(\mathrm{EVM}=18.1 \%)$ simplified SSBI compensation.

For the case of transmission over $480 \mathrm{~km}$ of SSMF, the performance of all $7 \mathrm{WDM}$ channels was measured at the optimum launch power per channel without and with simplified SSBI mitigation, as shown in Fig. 3(c). The average BER across the channels was reduced from $1.5 \times 10^{-2}$ to $3.8 \times 10^{-3}$. The net bit rate was $24 \mathrm{~Gb} / \mathrm{s}$ per channel based on the theoretical hard decision decoding bound for the binary symmetric channel at the BER of $3.8 \times 10^{-3}$, and the net optical ISD was $2.4(\mathrm{~b} / \mathrm{s}) / \mathrm{Hz}$.

\section{Conclusions}

We proposed a SSBI cancellation technique with low electronic hardware complexity for DD SSB SCM systems. Its back-to-back and transmission performance was experimentally tested on a $10 \mathrm{GHz}$-spaced WDM SSB 16-QAM NSCM system, operating at a net bit rate of $24 \mathrm{~Gb} / \mathrm{s}$ per channel (a net optical ISD of $2.4(\mathrm{~b} / \mathrm{s}) / \mathrm{Hz}$ ). To the best of our knowledge, this is the first experimental assessment of the simplified SSBI mitigation technique that combines the pre- and post-compensation and, consequently, avoids multiple iterations at the receiver DSP.

This work has been supported by the UK EPSRC UNLOC project, EU FP7 ASTRON project and Semtech Corporation.

\section{References}

[1] J.C. Cartledge et al., "100Gb/s intensity modulation and direct detection," J. Lightw. Technol., 32 (16), 2809-2814 (2013).

[2] A.O.J. Wiberg et al., "Single cycle subcarrier modulation," Proc. OFC, OTuE1 (2009).

[3] M.S. Erk1linç et al., "Spectrally-efficient WDM Nyquist pulse-shaped 16-QAM subcarrier modulation transmission with direct detection," J. Lightw. Technol., 33 (15), 3147-3155 (2015).

[4] W. Peng et al., "Spectrally efficient direct-detected OFDM transmission employing an iterative estimation and cancellation technique," Opt. Express, 17 (11), 9099-9111 (2009).

[5] Z. Li et al., "Signal-signal beat interference cancellation in spectrally-efficient WDM direct-detection Nyquist-pulse-shaped 16-QAM subcarrier modulation," Opt. Express, 23 (18), 23694-23709 (2015). 